\title{
Currículum flexible: estudiantes universitarios sorteando la conformación de su carga académica semestral
}

\author{
Elizabeth Ocampo-Gómez \\ Instituto de Investigaciones en Educación, Universidad Veracruzana. Lomas del Estadio s/n. C.P. 91000. Xalapa, Ver. \\ México (correo-e: eocampo@uv.mx)
}

Recibido Oct. 22, 2020; Aceptado Dic. 18, 2020; Versión final Ene. 7, 2021, Publicado Abr. 2021

\begin{abstract}
Resumen
El principal objetivo de este estudio es entender la interacción de los estudiantes con un establecimiento institucional de modelo flexible. Este es un estudio cualitativo con un diseño semi-estructurado que indaga cómo estudiantes universitarios con diferentes grados de avance crediticio sortean la conformación de su carga académica. Como resultado se identifican cuatro etapas sobre el procedimiento de selección de asignaturas: el candado. visualizar la oferta, la inscripción, y cursar las asignaturas. Los hallazgos vinculan el éxito de los estudiantes en la estructura de la carga semestral con: 1) su familiaridad con el modelo educativo, 2) su dominio de la burocracia universitaria, 3) su manejo de las plataformas digitales, 4) su acceso y conectividad al internet, 5) el acercamiento que tengan con el tutor o con compañeros de avance crediticio mayor, y 6) con el acogimiento institucional. Se concluye que la estructuración de la carga semestral implica cruzar las cuatro etapas referidas lo que complejiza la forma en la que los estudiantes transitan entre semestres.
\end{abstract}

Palabras clave: trayectorias estudiantiles; modelo universitario; flexibilidad curricular; tutorías académicas; experiencia escolar

\section{Flexible curriculum: university students facing their course- load configuration}

\begin{abstract}
The main objective of this research is to examine how students interact with an academic institution that uses a flexible model of education. This is a qualitative study with a semi-structural design that assesses how university students at different stages in their academic degrees manage to adapt their academic workloads. Four stages are identified: the padlock, seeing the offer, the registration, and taking the classes. These stages illustrate the required procedure for course selection. The results also show that there student course-load and school life success are linked to: 1) student familiarity with the educational model, 2) student understanding of academic bureaucracy, 3) student proficiency in handling digital platforms, 4) student internet access and connectivity, 5) student relationships with their academic advisor and with fellow students further ahead in their program, and 6) university welcoming and support. It is concluded that course-load planning involves facing the four stages mentioned above which challenge student advancement between semesters.
\end{abstract}

Keywords: student pathways; university model; curriculum flexibility; academic advising; school experience 


\section{INTRODUCCIÓN}

Este estudio fue realizado con el objetivo de conocer cómo experimenta la conformación de su carga académica semestral un grupo de estudiantes en una universidad de modelo flexible. A diferencia de la nutrida gama de investigaciones sobre estudiantes y trayectorias escolares (Castro-Montoya et al., 2020; Haas y Hadja, 2020), el presente trabajo no pretende tipificar ni a los estudiantes, ni su tránsito por las universidades, ni mirar los patrones de progresión (Cosser, 2018); tampoco aspira hacer relaciones de su trayectoria con la disciplina de su adscripción, sus orígenes socio-económicos, genero, etnicidad o desempeño académico (Martínez-Padilla y Pérez-González, 2008). Se trata de un trabajo cualitativo que busca abrir una ventana a las vivencias de un grupo pequeño de estudiantes universitarios en relación a la estructura organizativa que la Universidad Veracruzana ha dispuesto para que transiten de semestre a semestre.

En el libro publicado por el ANUIES, en el año de 2013, en el que se revisa el estado de la cuestión en temas de estudiantes en la primera década del siglo XX, Miller apunta que poco se sabe sobre la interacción de los estudiantes con el establecimiento institucional y sobre cómo las dinámicas organizacionales inciden en la formación académica (2013). Miller subraya que los estudios sobre trayectorias, en esa década, habían apenas comenzado a mostrar interés por conocer "Ios procesos escolares en los que se hallan involucrados los estudiantes y sus trayectorias y ponerlos en diálogo con otros eventos no académicos" (p. 183), apuntando que, hasta esa década, habían prevalecido las líneas clásicas del estudio de recorridos escolares que miraban las causas del rezago o abandono con el objetivo de documentar por qué no se lograban los ideales institucionales de continuidad.

Académicos como Mancera (2013) secundan la importancia de decantarse por nuevos enfoques de estudio de trayectorias apuntando que "...quizás sea de más provecho plantearse ya no sólo quiénes son los estudiantes sino cómo son, indagando en torno a los procesos, las acciones, las estrategias y las expectativas que éstos ponen en juego para transitar por la universidad..." (p. 52). Revisando la literatura, se pueden identificar algunos estudios cuyo objetivo ha sido entender cómo navegan los estudiantes, los requisitos, las normas, las expectativas, los tiempos y la burocracia institucional en modelos de educación flexible (Barrón y Díaz Barriga, 2016; Cosser y Nenweli, 2014; Drake, 2011; Giani, 2015), y cómo estos elementos inciden en su vida escolar, y personal, en sus decisiones (Musso, et al, 2020; Suhlmann, et al, 2018), emociones, intereses, y hasta en sus cuerpos (Dubet, 2009).

Desde que la noción de flexibilidad entró al campo educativo, ha estado envuelta en una multiplicidad de significaciones. En particular, el término "flexibilidad curricular" genera debates y cuestionamientos en torno a quién y para qué se flexibiliza (Bovill y Woolmer, 2018; Shay, 2013) o si de hecho se logra la flexibilidad (Nunes, 2019). Las autoridades educativas, desde su narrativa, presentan a la flexibilidad como una característica a la que toda institución debe aspirar. Lo anterior, debido a que se asume que la flexibilidad favorece la libertad de elección de asignaturas y trayectorias escolares, la autonomía estudiantil, la estructuración de perfiles académicos acordes a las aspiraciones profesionales de cada estudiante, la posibilidad de hacer ajustes en la carga académica de tal modo que se consideren los intereses y necesidades de cada estudiante (Díaz-Barriga et al., 2020; White y Schulenberg, 2012).

Aunque estos rasgos de la flexibilidad educativa son deseables (Ocampo et al, 2016), en la práctica no siempre se logran -como se muestra en este trabajo-. Para muchos estudiantes, la flexibilidad implica responsabilizarse por lo que "eligen", incluso cuando las opciones son magras y los cupos limitados. Muchos refieren sentirse constreñidos por estas circunstancias, y difícilmente creen los discursos oficiales sobre la flexibilidad. Para otros, la flexibilidad curricular significa que los horarios escolares pueden tomar casi cualquier espacio de su tiempo, incluyendo fines de semana, tardes, noches, horario laboral y horario otrora entendido como personal y separado de lo escolar (Ocampo et al, 2016).

Flexibilidad curricular conlleva, en muchos casos, sacrificar la posibilidad de trabajar, de socializar con amigos fuera del ámbito escolar, de dedicar tiempo a actividades extraescolares; para muchos representa interferencias en el desarrollo de sus intereses de juventud; también significa ofrecer plena disponibilidad para que la carrera universitaria tome casi cualquier horario de sus vidas. La flexibilidad que ofrece la universidad, en cierta forma representa la preparación hacia la flexibilidad laboral. Nunes (2019) explica, "flexibilizar el currículo significa crear estrategias para hacer los currículos más viables y más adecuados a las dinámicas de trabajo. Por tanto, se asocia a una percepción de empleabilidad, en la que se priorizan formaciones más pragmáticas con vistas a producir subjetividades coherentes como la producción y como la competitividad, características propias del paradigma de flexibilización, de hecho, neoliberal".

Aunque la flexibilidad laboral no es lo mismo que la flexibilidad curricular, el análisis realizado en este artículo, argumenta que hay paralelismos. Esto es porque que las condiciones para la construcción de trayectorias escolares en contextos de oferta reducida, ofrecen escenarios similares a los del campo laboral, produciendo un juego de rigidez-flexibilidad cargado de una gran ambigüedad tanto conceptual como práctica. Para autoras como Acacia Kuenzer (2017), la preparación para la flexibilidad laboral comienza en las instituciones 
educativas, mismas que, desde los años noventa, han revelado simpatía por el régimen de acumulación flexible. Desde la óptica de la autora, "el aprendizaje flexible es una nueva forma de mercancía que, para ser producida y consumida, demanda una formación de subjetividades flexibles: pragmatistas, situadas en el presente y fragmentadas." (Kuenzer, 2017, p. 348) [Mi traducción del portugués].

En este sentido, este trabajo busca dar voz a las vivencias de un grupo de estudiantes universitarios en un modelo de educación flexible. Como objetivos, se propone explicar la manera en la que estudiantes de trayectoria inicial, media y avanzada viven la conformación de su carga académica semestral, qué retos enfrentan, cómo los resuelven, cómo los interpretan, qué factores entran en juego. En tal análisis se identifican cuatro etapas que ilustran el procedimiento requerido para transitar de un semestre a otro. Como se explica en los apartados que siguen, cada etapa detona diferentes vivencias, retos, y reacciones de los estudiantes permitiendo que se aborden las principales categorías de análisis para responder a las interrogantes planteadas.

Estas etapas fueron definidas como: a) "el candado" -término elegido por los estudiantes para referirse al permiso para que se abra la plataforma de preinscripción que consiste en la evaluación docente de las clases del semestre previo, b) la visualización de la oferta académica donde los estudiantes realizan la selección de las asignaturas que les gustaría cursar, c) la inscripción, que incluye tanto la asignatura que se desea cursar y el profesor a cargo, como la asignación de un horario, y d) cursar las asignaturas, donde se describen algunas consecuencias que enfrentan los estudiantiles tras estructurar su carga crediticia.

Dichas etapas informaron de manera dialógica la metodología, el diseño de instrumentos, la selección de categorías de análisis, jugando un importante rol en el análisis de los resultados. Aunado a su valor metodológico y analítico, las etapas permiten presentar una investigación que focaliza los aspectos micro de la construcción de las trayectorias estudiantiles, y cómo es que éstos son mediados por aspectos meso -en este caso, la burocracia institucional en turno. En los resultados, sobresale que el éxito en la estructuración de la carga semestral está vinculado al desempeño de los estudiantes en tanto que los horarios iniciales para la selección de asignaturas son concedidos a estudiantes con mejor promedio, a su familiaridad con el modelo educativo flexible, su dominio de la burocracia universidad, su manejo de las plataformas digitales, su acceso y conectividad al internet, y al acogimiento institucional.

El cambio de la producción fordista a la toyotista introdujo el paradigma de la supuesta flexibilidad que mucho se ha diseminado como el rompimiento de estructuras inamovibles para dar cabida a la maleabilidad, la diversidad de alternativas; enfocando la visión en torno a lo que se requiere, en lugar de lo que se ofrece por sistemas preestablecidos (Au, 2011; Kuenzer, 2007). Este término se ha extendido hacia el campo de la educación, fomentando primeramente la diversificación de la oferta profesional (Kuenzer, 2007, p. 115) hasta llegar a las trayectorias individuales por sobre las grupales; el currículum por acumulación de créditos, en lugar de uno por tira de asignaturas preestablecida (White y Schulenberg, 2012). Díaz-Barriga, López-Ramírez y López-Banda apuntan que, hay muchos factores que intervienen -la gestión institucional entre ellos- en los eventuales beneficios de los modelos flexibles (p.7, 2020). Esta investigación se interesa en conocer cómo las características institucionales, pero también la estructura curricular y la oferta educativa inciden en las experiencias escolares enmarcadas en las narrativas de flexibilidad curricular.

Los estudios de currículo flexible y trayectorias estudiantiles tienden a abordar el nivel macro y meso de la política, tienden a ser de tipo cuantitativo mirando tipos de trayectorias, características sociodemográficas de los estudiantes (Haas y Hadja, 2019), patrones de progresión de poblaciones grandes (Cosser, 2018) para determinar el tiempo de permanencia, los egresos, las bajas, la reprobación de asignaturas, entre otras (Castro-Montoya, et al, 2020). Se trata de estudios que escasamente recuperan historias particulares para entender qué hay detrás de los índices estadísticos, cómo se construyen las historias particulares en torno a las experiencias de progresión por la universidad, bajo qué contexto se toman decisiones (Robinson y Bornholt, 2007). El estudio aquí expuesto mira hacia adentro de la institución educativa, ofrece un acercamiento con los estudiantes mostrando sus retos al navegar la burocracia institucional; de manera más cercana, explora su sentir, sus hábitos, sus estrategias para resolver problemas. Además, identifica etapas que suelen aparecer desdibujadas, tanto en los estudios de las trayectorias escolares, como en la propia normatividad universitaria. Bajo estas coordenadas, este estudio complementa lo que ya sabemos sobre los estudiantes en sus contextos escolares, con lo que sucede en etapas específicas de su tránsito entre semestres.

\section{METODOLOGÍA}

Esta investigación fue realizada en la Universidad Veracruzana (UV), en uno de los 178 programas de licenciatura que ofrece la universidad. Como parte del compromiso de resguardar la confidencialidad y datos de los participantes, no se expone información específica ni del programa estudiado, ni de los estudiantes que participaron en la investigación. 
La UV es una institución pública integrada por 62,115 estudiantes de licenciatura, 1,688 de posgrado y 465 de Técnico Superior Universitario (Universidad Veracruzana). Además de sus 178 programas de educación formal a nivel licenciatura, ofrece programas de educación no formal a los que se inscribe un gran número de estudiantes. Como universidad estatal, la UV ofrece estudios desde cinco regiones que abarcan las ciudades más importantes del Estado. A todas luces, se trata de una universidad con alcances, poblaciones, estructuras y liderazgos sumamente diversificados. Para los propósitos de este estudio, cabe resaltar que, en el año de 1999, la UV instauró un modelo de educación flexible al que se inscribieron de manera gradual todas las carreras que la componen, tomando 11 años para completar la transición.

A más de 20 años de haber emprendido su transformación curricular, la UV aún sigue consolidándose. En una evaluación general de su modelo se mostró que los resultados de trabajar con el modelo flexible son variados, con facultades donde el modelo es administrado de manera eficaz y benéfica para la mayoría de los involucrados, y otras en las que aún hay muchas oportunidades de mejora (Ocampo et al, 2016). El estudio aquí presentado únicamente refleja las opiniones de los estudiantes de una facultad; por consiguiente, lo que aquí se relata no necesariamente es representativo de lo que sucede en toda la universidad. Teniendo como objetivo responder preguntas de proceso y de vivencias, y de recuperar las narrativas sobre la experiencia, las visiones e interpretaciones de los estudiantes sobre su experiencia escolar en torno a la conformación de su carga semestral, se diseñó un estudio de corte cualitativo. En este estudio participaron un total de nueve estudiantes que fueron seleccionados mediante una muestra intencional que respondió al interés de documentar posibles variaciones sobre cómo viven la gestión escolar estudiantes con diferentes niveles de avance crediticio.

Participaron tres estudiantes de avance crediticio inicial que acababan de concluir su primer o segundo semestre; tres de avance medio que cursaban quinto o sexto semestre; y tres de trayectoria avanzada que estaban en noveno o décimo semestre. La selección de participantes se llevó a cabo mediante una invitación abierta, expuesta de manera verbal en grupos de clase donde se encontraban inscritos estudiantes de los grados de avance descritos. Tras hacer la invitación verbal en las clases donde así lo permitieron los docentes a cargo, dejamos oficios explicando con mayor detalle los objetivos y alcances de la investigación, lo que se requeriría de cada participante (en caso de que aceptaran contribuir) y una explicación de cómo se cuidarían los aspectos éticos de la investigación para proteger su identidad. Además de los datos generados con las entrevistas aplicadas, se recurrió a datos institucionales de carácter numérico de administración escolar, numeralia de la Universidad Veracruzana, evaluaciones sobre el Modelo Educativo de la Universidad Veracruzana.

Una vez obtenido el consentimiento voluntario de los participantes requeridos, se procedió a la realización de entrevistas previamente piloteadas con las que se afinaron los temas a estudiar. Las entrevistas siguieron un diseño semi-estructurado donde se delinearon temáticas a tratar, dando holgura para profundizar en temas no previstos relacionados con el objetivo de la investigación. En tanto que las indagaciones se volvieron más sofisticadas conforme se conversaba con más estudiantes, en algunos casos se acordó un segundo encuentro con los primeros entrevistados para explorar temas que habíamos omitido o que en los avances la investigadora detectó que podían ser explorados con mayor profundidad. Es importante destacar que todas las entrevistas fueron realizadas durante el año de 2018; éstas fueron grabadas en dispositivos móviles, teniendo cada una, en promedio, una duración de 40 minutos. Las transcripciones sirvieron como primera etapa de análisis y como una oportunidad para verificar las categorías establecidas. Una vez realizada la transcripción completa, se procedió a revisarlas usando el método de análisis de contenido (Berg, 2001).

Interesaba conocer cómo se alimentaban las categorías de análisis propuestas de manera inicial, qué otras categorías no contempladas habían emergido y cómo se relacionaban los resultados de cada categoría entre sí. Las categorías se plegaron a las etapas arriba mencionadas para transitar entre semestres, sin embargo, también se incluyeron sub-códigos, tales como efectos emocionales (estrés, decepción, frustración), efectos físicos (enfermedad, nutrición). Cabe resaltar que, respondiendo al compromiso de resguardar las identidades de los participantes, los nombres asignados a cada uno son pseudónimos.

\section{RESULTADOS}

Los resultados presentados intentan responder de manera puntual a cuestionamientos sobre los retos que enfrentan los estudiantes en la conformación de su carga académica semestral, y la forma en la que éstos interpretan el proceso de tránsito entre un semestre y otro. Para tal fin, esta investigación se apoya en los abordajes de la sociología de la experiencia escolar (Dubet, 2009), y de estudios del currículum flexible y trayectorias escolares (Barron, 2010; Bovill y Woolmer, 2018; Shay, 2013, Nunes, 2019).

\section{Cómo se estructura la carga académica}

Tras haber llevado a cabo el análisis de resultados, se identificaron los pasos requeridos para transitar entre semestres. A continuación, se sistematiza la opinión de los participantes dentro de cada paso, incluyendo un análisis enmarcado en las teorías arriba descritas. 


\section{El candado}

El término "candado" es utilizado de manera informal tanto por estudiantes como por autoridades educativas para referirse a un pre-requisito que, de no ser cumplido, impide que los estudiantes tengan acceso 0 procedan a navegar en las plataformas donde se visualiza la oferta educativa del siguiente semestre. En el año de 2015, la UV reportaba el uso de 21 candados implementados para asegurar que los usuarios se plegaran a políticas institucionales (Ocampo et al, 2016). Se trata de un "candado" que no se abre hasta que los estudiantes evalúan el desempeño de los profesores con quienes tomaron alguna clase en el semestre que concluyen. Aunque hay candados que cuidan la trayectoria estudiantil (como es el caso de los candados para las asignaturas seriadas o con pre-requisitos establecidos para evitar registros en cursos avanzados cuando no se han obtenido los conocimientos de cursos básicos), el candado aquí referido es un instrumento para evaluar el desempeño de los maestros.

La opinión de los estudiantes sobre el candado evidencia dos cosas: primero, la mayoría describe el candado como necesario en tanto el ejercicio de evaluación sirve como instrumento de mejora del quehacer docente. Por otro lado, otro tanto de estudiantes se enfoca en criticar el carácter coercitivo de un instrumento que no tendría porque ser parte de la gestión escolar que regula cómo transitar entre semestres (Wanda, 9o Semestre, p. 3; Gloria, 1er Semestre, p. 2). Se redactan dos de las variadas perspectivas compartidas por los estudiantes: "Yo pienso que está bien. Es muy tediosa [la evaluación docente], pero creo que es la única manera en la que obligas a que todos la hagan... es importante que te evalúen para saber cómo es tu [sic] desempeño" (Yanet, 5o Semestre, p. 2). "Pues yo pienso que está bien, porque bueno, muchas veces los maestros no se dan cuenta de las cosas en las que están mal hasta que ya tu [sic] como que le dices o le pones en la evaluación. Yo creo que sí [está bien que sea obligatoria], porque pues como que a muchos les da como flojera, pero está bien, porque el maestro luego falla en muchas cosas, ya tú se lo pones" (Hortencia, $5^{\circ}$ Semestre, p. 2).

Pero también hay quienes cuestionan el seguimiento que se le da a los resultados para que los docentes mejoren su práctica, como lo expresan estos estudiantes: "[...] no es así como que hagan una reprimenda al maestro, o no sé de qué manera se aplique para los maestros la lectura de las evaluaciones. Pero pues, como alumnos, obviamente queremos expresar sobre cómo fue el trato del maestro frente a nosotros, entonces a mí se me hace como necesaria" (Daniela, 5 Semestre, p. 2). "Yo creo que [...]que es una oportunidad tanto de nosotros como alumnos pues, para poder expresar lo que vivimos en lo que es dentro del aula con el maestro, la forma de enseñanza. También siento que si realmente se toma en cuenta, pues está bien, porque, pues el docente puede mejorar de ahí a partir de la evaluación" (Eva, 9o Semestre, p. 3). "La evaluación es una cosa para ver en qué estás fallando y si puedes mejorar, pero la mayoría de las veces no se hace nada [...] no cambia nada, no se mejora, no se hace nada por la evaluación" (Alberto, $7^{\circ}$ Semestre, p. 2).

Como podemos ver, los cuestionamientos son expresados por estudiantes con un mayor grado de avance semestral, poniendo en juicio la efectividad del instrumento y la participación obligatoria. Algo que nadie cuestiona, quizás por desconocimiento, es que el puntaje de las evaluaciones de los maestros está ligado al programa de incentivos salariales de la UV. Este vínculo es un mecanismo de gestión muy práctico en tanto que obligar a los estudiantes a evaluar a sus profesores contribuye a que los profesores aspiren a un mejor sueldo. No recibir evaluaciones, hace que los profesores se vean impedidos de participar en los programas de incentivos de la universidad. Ahí reside la importancia del mecanismo de evaluación obligatoria. Sin embargo, su carácter coercitivo y la poca o nula evidencia de que las evaluaciones sirvan para mejorar la práctica docente, desplaza los alcances del instrumento hacia lo preponderantemente administrativo circunscribiendo el beneficio únicamente al profesorado.

Los estudiantes no tardan muchos semestres en significar al candado como algo que puede ser removido contestando los cuestionarios con poco cuidado y la mayor velocidad posible para poder ver la oferta del siguiente semestre. Cabe señalar que el instrumento se contesta la cantidad de veces que hayan tomado asignaturas, lo que añade al tedio y desinterés de los estudiantes. Bajo estas consideraciones es menester preguntarse si no es un paso que puede trasladarse a otro momento que no sea el tránsito entre semestres, agilizando así el trayecto estudiantil y quizás recuperando el valor de un instrumento con potencial para incidir en la calidad docente.

\section{Visualización de la oferta educativa}

Esta etapa, también conocida como el periodo de preinscripción semestral, es un ejercicio de planeación curricular en el que se proyecta la ruta del siguiente semestre con la ayuda de plataformas digitales, y de manera ideal con el acompañamiento de un tutor académico. Los estudiantes seleccionan asignaturas que quieren y pueden cursar, también visualizan si cumplen con pre-requisitos (cuando es el caso) en asignaturas que desean cursar, y de acuerdo a lo mostrado, pueden tomar decisiones. Los tutores académicos, 
responsables de conocer el plan de estudios y las áreas del modelo, pueden brindar orientación y responder dudas respecto a la selección de las rutas curriculares del programa. Cabe señalar que el acompañamiento tutorial para la construcción de trayectorias estudiantiles ha estado presente desde 1999 cuando se implementó el modelo flexible en la UV; sin embargo, su operación se ha vuelto más sofisticada gracias a la implementación y mejora de plataformas digitales.

La pre-inscripción tiene como función optimizar recursos, asegurarse de que no se abran clases con pocos estudiantes, o que las clases con demasiada demanda se puedan dividir en secciones. En currículos flexibles, este tipo de estrategia es ineludible en tanto que la apertura mal planeada puede resultar costosa para la universidad y para estudiantes cuyas necesidades curriculares no se ven cubiertas, haciéndolos aplazar el curso de todos sus créditos para egresar. En cierta medida, la visualización de la oferta entusiasma a tutores responsables, pero sobre todo a los estudiantes de semestres iniciales, ya que visualizan, sin prisas y sin restricciones, la oferta completa del semestre por comenzar. La opinión de los estudiantes al respecto se avocó a describir que revisan la oferta, se familiarizan con las asignaturas que abrirán para saber qué asignaturas registrar, y luego hacen su planeación. Varios refirieron hacer este ejercicio con el acompañamiento de los tutores (Gloria, 1er Semestre, p. 3; Hortensia, 1er Semestre, p. 2).

Con frustración, una estudiante comenta que, de varios horarios tentativos, en la etapa de inscripción no puede elegir ni uno: "sí, siempre hago como diez horarios diferentes. Al final no puedo meter ninguno." (Yanet, 5o Semestre, p. 2). Otros también comparten su opinión sobre el rol que desempeña el tutor en esta etapa, haciendo críticas que enfatizan la necesidad de una labor tutorial más comprometida. El siguiente extracto es un ejemplo de lo anterior: "Sí, porque cuando tuvimos la tutoría, o disque tutoría, hubo varios compañeros a quienes les dieron un como folder y en la parte de atrás venían como por colores las cadenas, una de color verde, azul, amarilla, y así, por eso sé que hay secuencia, pero así que me lo hayan explicado como tal, a mí no" (Gloria, 1er Semestre, p. 5).

Otros describen cómo compañeros con mayor avance crediticio hacen labores tutoriales que, dada su experiencia y acercamiento con los pares, puede resultar de mucha ayuda (Bruno, 1er Semestre, p. 5). Como ejemplo concreto del rol de los pares, una estudiante comparte: "Creo que fue nuestra tutora la que nos explicaba más o menos cómo se hacía ese proceso, igual entre los compañeros, entre los que ya eran de semestres más adelantados, y, pues, ya tenían esa experiencia ya nada más nosotros les preguntábamos." (Eva, 9o Semestre, p. 5). Pero en general, la mayoría describe tener tutores con quienes se reúnen y de quienes reciben orientación e incluso intervenciones burocrático-administrativas cuando es requerido, como la descripción del estudiante Alberto ( $7^{\circ}$ Semestre). Cabe apuntar que la ilusión de la pre-inscripción, que permite visualizar la oferta completa y hacer un ensayo de selección de la carga semestral para el próximo semestre, llevada a cabo con o sin los tutores, se esfuma cuando se abre la plataforma con las asignaturas que de facto se ofrecerán. Esto es explicado en la descripción de la siguiente etapa.

\section{La inscripción}

La inscripción sigue dos pasos, el primero sucede cuando la facultad asigna un día y un horario para que se inscriban los estudiantes, y el segundo cuando éstos llevan a cabo la selección y el acomodo de asignaturas. Aproximadamente dos semanas antes de que inicie el semestre, los secretarios de facultad informan a los estudiantes la fecha y la hora en las que podrán hacer la selección de las asignaturas definitivas a cursar. Este ya no es un ejercicio hipotético de lo que podría registrarse; es el registro definitivo. La distribución de tiempos de inscripción se lleva a cabo para no saturar un sistema con más de 60 mil usuarios al semestre, pero es también un procedimiento que toma en cuenta que no habrá cupo para todos en asignaturas de alta demanda, que en las asignaturas con más de una sección hay maestros y horarios que atraen a audiencias mayores, y que la oferta no es suficiente. Por lo tanto, la referida asignación es una herramienta para asegurar que funcione la plataforma, pero también sirve para controlar los ingresos en una oferta limitada, y como proceso de selección que en cierta medida acentúa las disparidades entre estudiantes (Giani, 2016) resultando en un instrumento de meritocracia que beneficia a estudiantes con mejor desempeño.

Los relatos sobre esta etapa refieren que ésta se vive con ansiedad, estrés, desilusión, frustración (Daniela, 50 Semestre, p. 3; Wanda, 9 Semestre, p. 4; Gloria, 1er Semestre, p. 6) cuando se asignan horarios tardíos en los que ya quedarán pocas alternativas. Se recuperaron varias opiniones al respecto, por cuestiones de espacio solo se comparte la más representativa: "Pues me pongo nerviosa...ya tengo en mente las materias que voy a meter, pero también me pongo así como, ¿y si no me tocan?, ¿y si me las ganan?, o ¿si no las ofertan?, o ¿si chocan los horarios? Entonces, como que es mucho nervio, mucha tensión de estar pensando qué va a pasar en el momento en que te estás inscribiendo" (Eva, 9올estre, p. 4).

Los estudiantes de primer semestre no eligen asignaturas. En lugar de esto, argumentando que primero deben familiarizarse con el modelo flexible, algunas facultades arman -con toda rigidez - la carga de los semestres 
iniciales. Por tal motivo, los estudiantes entrevistados que habían elaborado su carga por primera vez, comentaron que no entendían completamente la dinámica, haciendo alusión a lo "injusto" que les parecía que a unos les tocará inscribirse antes que a otros (Hortensia, 1er Semestre, p. 3; Bruno, 1er Semestre, p. 3). Con relación a los criterios que se usan para asignar horarios específicos, también se evidenció que muchos estudiantes tienen poca claridad sobre los criterios que se usan para la asignación de horarios. Cuando se les preguntó sobre este tema, hubo muchas especulaciones, pero nadie supo con certeza cuáles eran los criterios: "No, o sea, hay como mitos, que es por apellido, que es por semestre, que es por avance crediticio, que es por promedio, que no sé...miles de formas que no... incluso que es aleatorio, que es al azar, pero pues nunca he sabido... de hecho, no creo que nadie sepa cómo se hace...me temo que es por el promedio" (Alberto, $7^{\circ}$ Semestre, p. 3)

El segundo paso de esta etapa consiste en seleccionar y acomodar los horarios de las asignaturas deseadas. Aquí entran en juego otras variables. Por ejemplo, hay asignaturas con cupo disponible, que le conviene al estudiante registrar y que abonan al perfil profesional deseado; sin embargo, tienen los mismos horarios que otras asignaturas ya seleccionadas. Es decir, son asignaturas que "chocan", según las palabras de los

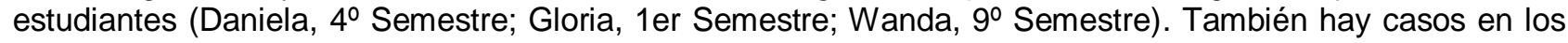
que los alumnos identifican asignaturas interesantes, con cupo, pero con horarios muy alejados entre sí, que

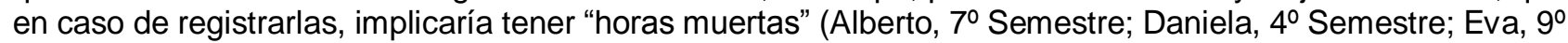
Semestre; Wanda, 9 Semestre). Las "horas muertas" son horas sin clases, en las que los estudiantes no pueden regresar a sus casas por lo que implicaría en costos y tiempo de traslado, ni pueden trabajar por lo fragmentada que quedaría su disponibilidad.

Así, elaborar el horario deseado implica tomar en consideración todas estas variables, como cuenta este participante: "Sí, me ha pasado muchas veces, pues, ya no sé. O sea, tú [sic] ya tienes planteado un horario, ya sabes qué vas a hacer, y luego te choca con otra, y entonces sacas como tres materias para meter dos, pero son como las que más te importan, y al final no sirve de nada haber hecho tu planeación porque, con una que te falte o con una que te choque, se echa a perder todo" (Alberto, $7^{\circ}$ Semestre, p. 4). Otra estudiante añade información sobre las complicaciones que enfrentan: "Nada más hay tantos lugares, tantos maestros, y si ya se cerró el grupo entonces tú [sic] quedas fuera, ¿no? Y pues es la materia que tú [sic] también necesitas...[Yo] buscaba, o sea, no cambiaba yo mi horario. Por decir, si ya me quitaron una materia, pues lo que hacía era buscar otra que me hacía falta y que podía yo acomodar" (Eva, 9 Semestre, p. 4)

Los estudiantes se vuelven gestores no solo de su carga académica, sino de plataformas virtuales, de lo imprevisto y de la escasa oferta, deben entender las normas institucionales (Suhlmann, et al, 2018) y la lógica administrativa de la institución. Robinson y Bornholt (2007) sugieren que cuando se estudien las trayectorias estudiantiles, se considere el contexto en el que suceden las elecciones. En esta universidad se identifica un contexto en el que gran parte de lo descrito gira en torno a la gestión y a los recursos. Los estudiantes, con la amplia variabilidad de recursos o características que tengan, se ajustan a una gestión y oferta que es más rígida que flexible. Sobre la experiencia que tienen acerca de las plataformas virtuales, encontramos que hacen referencia a la incertidumbre que sienten sobre si funcionará o no su conectividad a la red, motivo por el que algunos prefieren buscar lugares aparentemente más confiables para realizar su inscripción a través de Internet.

De lo anterior nos habla el siguiente estudiante: "Porque se me hace más confiable que en mi casa, porque luego puede fallar o la luz se desconecta o cualquier accidente, y me altero o no sé qué hacer, y ya en un ciber es como, bueno... yo llego y por obligación, tiene que estar el Internet conectado". Al preguntarle a dicho estudiante por qué existe el temor a que algo falle, respondió: "Porque he escuchado de compañeros que sí les ha pasado, que han tenido accidentes, que se les cae la computadora, se les apaga el celular, o que se les bloquea lo que sea y, a la mera hora, mal" (Alberto, $7^{\circ}$ Semestre, p. 4)

La decepción, la ansiedad, el estrés y la frustración son sentimientos que abundan en las narrativas de los estudiantes que describen cómo es su inscripción. En este trabajo se recuperaron relatos sobre cómo la necesidad de estructurar una carga de créditos adecuada para el semestre, se hace poco factible. Los estudiantes no ven ofertadas las asignaturas que necesitaban. En casos de reprobación los estudiantes deben registrarse en "segunda oportunidad" pero no siempre hay oferta o la oferta se satura por la prioridad a los estudiantes con promedios altos. Esto hace que en ocasiones deban esperar uno o dos semestres para volver

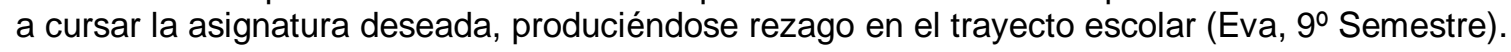

Los estudiantes describen con vehemente desencanto cómo lo que visualizaron durante la pre-inscripción, termina siendo diferente a lo que lograron en la inscripción. Sospechan que las asignaturas con las que "se quedaron" estarán poco vinculadas con el perfil profesional que deseaban conformar. Sus tutores o compañeros les sugieren que, si desean terminar su carga académica a tiempo, consideren la oferta de otras facultades o que registren asignaturas de elección libre que se vuelven comodín para estructurar una carga 
aceptable. Pero los créditos de elección libre son limitados, por lo que varios estudiantes los agotan mucho antes de terminar la carrera.

Tomar asignaturas de otras facultades en una universidad tan descentralizada, implica desplazarse a otras localidades de la ciudad usando los pocos recursos que tienen a su disposición los estudiantes de clase media-baja. Por lo tanto, las oportunidades de movilidad tienden a ser excluyentes, acentuando así desigualdades en un sistema que plantea dejar la libertad de elección en las manos de todos. Sobre este tema Dubet François explica cómo los modelos que asocian una estructura y un sistema escolar abiertos, en la práctica "engendran la exclusión". (Dubet, 2009, p. 2002).

\section{Cursar las asignaturas}

Cursar las asignaturas, más allá del reto de aprender contenidos, implica cumplir con horarios, el tiempo de permanencia diaria en la institución y la espera entre clases. Con base en las indagaciones con los estudiantes, se identificaron cuatro categorías de análisis que enmarcan los principales temas que surgieron. Estos temas se refieren a efectos de la carga académica en lo físico, lo emocional, los calendarios personales y los aspectos económicos. Se proveen los detalles de cada tema a continuación.

\section{Aspectos físicos}

Con relación a los efectos en lo físico, se encontraron relatos sobre la alimentación, el desgaste mental y el cansancio. Como tema común, en esta categoría de análisis sobresalen relatos de estudiantes que asisten a la universidad en ayunas. Las causas enunciadas hacen alusión a prisa matutina, descuido o desatención a esta necesidad o rutina. Por ejemplo, una estudiante comenta: "[...] ah, eso sí, no desayuno, porque no acostumbro a hacerlo tan temprano, [...]" (Hortensia, 1er Semestre, p. 7). En tanto que el ayuno suele ser prolongado $\mathrm{o}$ atendido con comida no nutritiva, esto llega a causarles malestares, tal como lo explica la siguiente estudiante: "Como problemas, creo que vienen las enfermedades, ¿no? Al menos en mi caso sí era de enfermarme mucho del estómago porque no tenía una buena alimentación [...]" (Eva, 9o Semestre, p. 8).

Para indagar más sobre este tema, en las entrevistas se incluyeron algunas preguntas sobre el contenido de sus mochilas durante su jornada escolar diaria. Al respecto, respondieron que llevan material escolar, agua, cartera, computadoras portátiles (algunos) y pertenencias de cuidado personal; solo dos describieron llevar comida. Lo anterior fue complementado por varios estudiantes que hicieron referencia a periodos largos de ayuno y consumo de comida poco nutritiva. Por ejemplo, uno de ellos comparte qué es lo que come: "Pues huevos, igual cualquier comida sencilla que no necesite tiempo, o compramos pizza, cualquier cosa basura, nunca comemos así como que bien." (Alberto, $7^{\circ}$ Semestre, p. 10). Otro aspecto sobresaliente es el desgaste físico, principalmente asociado a la permanencia prolongada en la facultad, al trabajo en las horas de clase y fuera de éstas. Este tema es abordado a mayor profundidad en los siguientes apartados.

\section{Aspectos emocionales}

En el aspecto emocional, se encontró que la capacidad de estructurar buenos horarios y una carga académica que responda a los intereses de los estudiantes, juega un papel importante en el despliegue de sus sentimientos y actitudes para afrontar el semestre. Por ejemplo, de los entrevistados, una estudiante expresa el sentimiento de agrado porque pudo coincidir con una amiga en algunas de las asignaturas, de igual manera porque eligió al docente con el que quería cursarlas (Hortensia, 1er Semestre, p. 3). El conformismo en los estudiantes se manifiesta cuando no logran inscribirse a las clases que eran de su interés y que planeaban cursar para el siguiente semestre. Como consecuencia de esto, simplemente reelaboran su horario con cualquier asignatura que les permita cumplir con la carga de materias obligada: "Sí, aunque no quedó como yo quería, pero pues ya qué puedo hacer. Pues, yo tenía una hoja en donde, según yo, realicé mi horario conforme a las materias que quería llevar o cursar este semestre, pero pues no quedó tal cual lo elaboré en mi borrador porque ya no estaban las materias, porque ya se habían acabado, pero pues de las que aún estaban, pues metí de esas" (Hortensia, 1er Semestre, p .4).

La frustración se manifiesta cuando los estudiantes están estructurando su horario y observan la dispersión de horas. Sobre esto se expresa una estudiante: "Me frustraba porque era mucho desfase de horas" (Wanda, 9o Semestre, p. 4). El desfase de horas puede crear un sentimiento de desilusión en los estudiantes, mismo que posiblemente repercute en sus trayectorias y en sus desempeños. Por ejemplo, otra estudiante comenta sobre los factores que le afectan emocionalmente: "Pues este...que se me llenaran las materias, que no pudiera agregarla a mi horario porque chocaban y tuve que cambiar todo completamente, me quedó el horario mixto...padecí horriblemente en ese momento porque se me cerraban todas, y pues ya no, ya no tenía ni cupo ni nada, y fue así como que entré en depresión, angustia, miedo y coraje" (Daniela, 5은 Semestre, p.8). 
Este tipo de repercusiones deben ser conocidas por tomadores de decisiones, autoridades que definen criterios de asignación de recursos, directivos y, sobre todo, secretarios de facultad cuyo rol es estructurar la oferta. Lo anterior, en tanto que el resultado de su trabajo administrativo tiene secuelas profundas en los estudiantes -y muy probablemente en los indicadores de la universidad-como se ha venido documentando aquí, que muchas veces pasan desapercibidas. Una propuesta pedagógica flexible que argumenta poner en el centro a los estudiantes, debe ser congruente en su operación (Nunes, 2019).

\section{Calendarios personales}

En cuanto a los calendarios personales, se encontró que, con excepción de las horas que utilizan para dormir, los estudiantes cuentan con poco tiempo libre dentro y fuera de la institución. Y aquellos que disponen de tiempo libre entre clases, lo utilizan para descansar, para hacer tareas, para comer o trasladarse a casa o a otras facultades. He aquí una opinión al respecto: "Mis beneficios podrían ser que al tener el tiempo suficiente, puedo descansar y puedo comer, pero, toda la semana así, durante un semestre, es cansado" (Ernesto, $7^{\circ}$ Semestre, p. 6). Llama la atención, sin embargo, cómo muchos estudiantes pasan su juventud en la institución educativa, reduciendo su tiempo para desenvolverse en otras áreas de su vida. Suárez Zozaya (2017) opina que las instituciones, sobre todo las de clase alta, en buena medida se convierten en el espacio de convivencia porque tienen las condiciones para acoger a los estudiantes, hay clubes gimnasios, asociaciones, cafeterías formalmente establecidas, espacios de recreación, jardines.

Sin embargo, esto no es así en instituciones públicas como la UV. Más específicamente, la autora comenta que: "La escuela total corresponde a la oferta educativa para la élite privilegiada, permitiendo una inmersión total de los jóvenes en la vida escolar y contribuye a la formación de una identidad estudiantil densa. En este caso, ser alumno universitario implica contar con un espacio de acogida para el desarrollo de prácticamente todas las actividades de la vida cotidiana, además de la dedicadas al estudio: hacer deporte en instalaciones seguras y adecuadas; convivir con amigos en restaurantes, cafés y jardines; conectarse a Internet en cualquier lugar del campus, practicar actividades artísticas, asistir a espectáculos culturales, etc" (Suárez Zozaya, 2017).

En el caso de instituciones públicas como la UV, los estudiantes refieren que el modelo flexible no propicia que conozcan y convivan en planos de amistad más certeros. Tienen encuentros muy efímeros y pocas veces vuelven a coincidir con quienes comienzan a desarrollar empatías. Cabe subrayar que en la facultad analizada no hay instalaciones deportivas para ejercitarse. Los lugares donde los estudiantes interactúan al salir del aula son una explanada, los puestos de comida (en su mayoría callejeros), una cafetería pequeña, el negocio de papelería, los pasillos, la biblioteca y el cubículo estudiantil. Además, hay muy poca infraestructura para sentarse, descansar o hacer tarea debido a que, cuando no hay clase, se cierran las aulas, dejando únicamente espacios como el piso de los pasillos o los escalones para sentarse.

La poca convivencia con los compañeros, y la escasa socialización de los estudiantes, se traslada también hasta sus hogares, en tanto que los alumnos salen de sus casas en la madrugada y regresan de noche, teniendo pocas horas para convivir con familiares o amigos cercanos, generando un aislamiento en sus vidas (Yanet, $5^{\circ}$ Semestre, p. 11). En general, las descripciones sobre los calendarios personales hablan de cómo las labores escolares y el tiempo de permanencia diaria acaparan el horario de los jóvenes. Esto no sólo los obliga a dedicarse de tiempo completo a la escuela, sino que, una vez inmersos en sus estudios, la institución tampoco hace mucho por brindar el acogimiento o la integración necesaria, documentada como crucial en la literatura que estudia cómo transitan los estudiantes por la universidad (Dubet, 2005; Giani, 2016; Leese, 2010).

Casillas, y colegas, en su artículo sobre el trabajo que realizan los estudiantes universitarios, explican que "Las instituciones de educación superior generalmente hacen caso omiso de las responsabilidades sociales que pueden tener sus estudiantes más allá del compromiso escolar. Sin embargo, existen algunos que trabajan, tienen dependientes económicos e, incluso, hijos" (Casillas, el al., 2001, p.3). Según estos autores, ignorar las responsabilidades extra-escolares de los estudiantes, deviene problemas tales como deserción, malestar estudiantil o bajo rendimiento escolar.

\section{Aspectos económicos}

El aspecto económico estuvo presente en el discurso de todos los estudiantes entrevistados. Los participantes relataron que estar de tiempo completo en la universidad complica su situación monetaria, porque no pueden trabajar y les genera gastos considerables (Alberto, $7^{\circ}$ Semestre; Daniela, $4^{\circ}$ Semestre; Ernesto, $7^{\circ}$ semestre; Eva, 9o Semestre; Gloria, 1er Semestre; Wanda, 9 Semestre). Robinson y Bornholt (2007) coinciden en que los factores financieros inciden en las posibilidades de elección y éxito académico de los estudiantes. La UV es una universidad pública a la que asisten muchos estudiantes de bajos recursos, para quienes ya de por sí 
los gastos de transporte representan retos considerables. En una evaluación del MEIF se describe cómo la movilidad es limitada por la capacidad económica de los estudiantes, donde $46.6 \%$ de los encuestados refiere que los gastos de la movilidad entre facultades de la misma ciudad rebasan sus posibilidades económicas (Ocampo et al., 2016). Los gastos de alimentación también son considerables, sobre todo en los estudiantes que salen de sus hogares sin desayunar y que no suelen llevar alimentos de casa (Daniela, 5 o Semestre, p. 7; Alberto, $7^{\circ}$ Semestre, p. 8). Lo anterior nos muestra que las condiciones económicas se vuelven un factor determinante para que los alumnos puedan beneficiarse o no de un modelo flexible con supuesto enfoque en los estudiantes.

\section{DISCUSIÓN FINAL}

Este estudio se propuso mostrar cómo son las dinámicas de interacción entre estudiantes y su universidad durante su tránsito entre semestres. En los resultados se hizo evidente que la administración educativa juega un papel importante cuando se quiere entender aspectos particulares sobre la construcción de trayectorias escolares. Los participantes subrayaron que el logro de la estructuración de trayectorias deseadas es mediado por su desempeño, pero también por su familiaridad con el modelo educativo flexible, su dominio de la burocracia universitaria, su manejo de las plataformas digitales, su acceso y conectividad al Internet, por el acogimiento institucional y por las tutorías de maestros y compañeros con experiencia en la universidad estudiada.

Autores como Suhlmann y colegas (2020), refieren que el acogimiento institucional contribuye a desarrollar el sentido de pertenencia explicando que "el sentido de pertenencia es una variable clave en la promoción del bienestar psicológico de los estudiantes, su motivación y en evitar que éstos se formen la intención de darse de baja" [Mi traducción del inglés] (p. 3). En tanto que la eficiencia terminal es uno de los principales indicadores de calidad, es menester hacer estudios como éste que focalizan la atención en aspectos micro a veces subestimados- de la trayectoria estudiantil.

El principal aporte de este estudio ha sido mostrar las complejidades que subyacen al estudiar aspectos puntuales de las trayectorias escolares. Con este trabajo, se evidencian necesidades apremiantes de los estudiantes que generalmente son ignoradas por los funcionarios y por hacedores de políticas. Este estudio, a su vez, enfatiza que es menester tener continuos acercamientos con los estudiantes para conocer en qué medida la administración universitaria impacta sobre aspectos particulares de sus trayectorias académica. El acogimiento institucional es un tema que permea buena parte de las percepciones de los estudiantes. Al verse inmersos en la institución de tiempo completo, los estudiantes desean mejores condiciones escolares, mismas que pueden incluir desde la infraestructura física necesaria y básica para practicar deporte, convivir y descansar, hasta un personal administrativo y docente más amigable y respetuoso de su tiempo y sus recursos, una burocracia y administración más consciente de sus necesidades; en resumen, una institución que propicie en ellos la tan deseada y documentada integración (Castro-Montoya, et al, 2020; Leese, 2010; Suhlmann, et al, 2018). Cabe destacar que la reacomodación de rutinas producida por las políticas de confinamiento derivadas del Covid-19, ha modificado temporalmente muchos de los elementos aquí descritos. Sabiendo que las condiciones que estamos atravesando serán temporales, interesa proveer insumos para corregir las tensiones identificadas en este trabajo.

\section{CONCLUSIONES}

De acuerdo al trabajo presentado y a los resultados obtenidos, se pueden plantear las siguientes conclusiones principales: 1) la estructuración de la carga semestral implica cruzar cuatro etapas que complejizan la forma en la que los estudiantes transitan entre semestres; 2) estudiar la experiencia escolar en relación a estas cuatro etapas, brinda una mirada específica a aspectos puntuales de la conformación de la carga de asignaturas y a las vivencias estudiantiles en relación al proceso necesario para avanzar entre semestres, da cuenta del peso que tiene la administración, los recursos y la burocracia educativa en la conformación de las trayectorias estudiantiles; 3) recuperar las narrativas de los estudiantes sobre sus experiencias en la conformación de su carga semestral, evidencia que éstos enfrentan retos considerables que van desde lo emocional y lo físico, hasta lo económico, y lo social.

\section{REFERENCIAS}

Au, W., Teaching Under the New Taylorism: High-stakes Testing and the Standardization of the 21st Century Curriculum, https://doi.org/10.1080/00220272.2010.521261, J. of Curriculum Studies, 25-45 (2011).

Barrón-Tirado, M.C., y Díaz-Barriga, F., Curriculum Management and the Role of Curriculum Actors, doi: https:doi.org/10.14288/tci.v13i2.188285, Transnational Curriculum Inquiry 13 (2), 13-33 (2016).

Berg, L. B., Qualitative Research methods for the social sciences, Allyn and Bacon, Boston, EUA (2001). 
Bovill, C., y Woolmer, C., How Conceptualisations of Curriculum in Higher Education Influence Student-staff Cocreation in and of the Curriculum, https:doi.org/10-1007/s10734-018-0349-8, Higher Edu. 78, 407-422 (2018).

Casillas, M. A., y otros tres autores., Los Estudiantes de la UAM-A, un Sujeto Social Complejo. Revista Mexicana de Investigación Educativa. ISSN: 1405-6666 6 (11), 139-163 (2001).

Castro-Montoya, B. A., y otros tres autores, Trayectoria Académica y Factores Asociados a Graduación, Deserción y Rezago en Estudiantes de Programas de Pregrado de una Universidad Privada de Medellín (Colombia), https:doi.org/10.4067/S0718-50062020000100043, Formación Universitaria, 13(1), 43-54 (2020).

Cosser, M., Differential pathways of South African Students through Higher Education, https:doi.org/10.1016/j.jjedudev.2017.10.003 59, Int. J. of Educational Development, 59, 100-109 (2018).

Cosser, M., y Nenweli, S., We've got you Pegged: Programme Choice in the Transition to, and Passage through, Higher Education, https:doi.org/10-1007/s10734-013-9655-3, Higher Edu., 67(3), 333-348 (2014).

Díaz-Barriga, F., López-Ramírez, J. L., y López-Banda, E. A., Trayectorias Personales de Aprendizaje y Currículo Flexible: La Perspectiva de los Estudiantes Universitarios de Psicología, https:doi.org/10.22201/iisue.20072872e.2020.30.585, Revista Iberoamericana De Educación Superior, 11(30), 3-21 (2020).

Drake, J., The Role of Academic Advising in Student Retention and Persistence, https:doi.org/10.1002/abc.20062, About Campus, 16 (3) 8-12 (2011).

Dubet, F., Las Paradojas de la Integración Escolar, ISSN: 1515-9485, Espacios en Blanco, Revista de Educación, 19 (19), 197-214 (2009).

Giani, M. S., The Postsecondary Resource Trinity Model: Exploring the Interaction between Socioeconomic, Academic, and Institutional Resources, https:doi.org/10.1007/s11162-014-9357-4, Higher Edu. 56(2), 105-126 (2015).

Giani, M. S., Are all Colleges Equally Equalizing? How Institutional Selectivity Impacts Socioeconomic Disparities in Graduates's Labour Outcomes, https:doi.org/10.1353/rhe.2016.0013, The Review of Higher Edu. 39(3), 431-461 (2016).

Haas, C., y Hadjar, A., Students' Trajectories through Higher Education: a Review of Quantitative Research, doi: 10.1007/s10734-019-00458-5, Higher Edu. 79, 1099-1118 (2020).

Kuenzer, A. Z., Trabalho e Escola: a Flexibilização do Ensino Médio no Contexto do Regime de Acumulação Flexível, https:doi.org/10.1590/ES0101-73302017177723, Educação \& Sociedade, 38 (139), 331-354 (2017).

Kuenzer, A. Z., Da Dualidade Assumida à Dualidade Negada; o Discurso da Fexibilização Justifca a Inclusão Excludente, https:doi.org/10.1590/S0101-73302007000300024, Educação e Sociedade. 28 (100), 1153-1178 (2007).

Leese, M., Bridging the Gap: Supporting Student Transitions into Higher Education, https:doi.org/10.1080/03098771003695494, Journal of Further and Higher Education, 34(2), 239-251 (2010).

Mancera, E., Características Socioeconómicas, Familiares y Laborales de los Estudiantes; en Estudiantes, Maestros y Académicos en la Investigación Educativa. Tendencias, aportes y debates, 2002-2011 de C. Saucedo, C. Guzmán, E. Sándoval, J. Gálaz, pp. 37-59., 2002-2011. ANUIES, México D.F., México (2013)

Martínez-Padilla, J. H. y Pérez-González, J. A., Efecto de la Trayectoria Académica en el Desempeño de Estudiantes de Ingeniería en Evaluaciones Nacionales, https:doi.org/10.4067/S0718-50062008000100002, Formación Universitaria, 1(1), 3-12 (2008).

Miller, G. D., El Estudio de las Trayectorias Escolares en México: un Aporte para el Nuevo Milenio; en Estudiantes, maestros y académicos en la investigación educativa. Tendencias, aportes y debates, 2002-2011 de C. Saucedo, C. Guzmán, E. Sándoval, J. Gálaz, pp. 183-197 ANUIES, México D.F., México (2013)

Musso, M.F., Hernández, C.F.R. \& Cascallar, E.C., Predicting key educational outcomes in academic trajectories: a machine-learning approach, https://doi.org/10.1007/s10734-020-00520-7, High Educ 80, 875-894 (2020).

Nunes J.A., O Discurso da (In)Flexibilidade Curricular em Análise Dialógica, https:doi.org/10.1590/1982-4017-1901064018, Linguagem em (Dis)curso, 19 (1), 87-105. (2019).

Ocampo Gómez, E., y otros seis autores, Formación Integral, Transversalidad y Flexibilidad en el Modelo Educativo de la Universidad Veracruzana. https://www.uv.mx/ (2016).

Robinson, R.A., y Bornholt, L.J., Pathways Theory of Progression through higher education, ISSN: 1446-5442 Australian J. of Educational \& Development Psychology, 7, 49-62 (2007).

Shay, S., Conceptualizing Curriculum Differentiation in Higher Education: a Sociology of Knowledge Point of View, https:doi.org/10.1080/01425692.2012.722285, British J. of Sociology of Edu. 34(4): 563-582 (2012).

Suhlmann, M., y otros tres autores, Belonging mediates effects of student university fit on well- being, motivation, and drop-out intention, https://doi.org/10.1027/1864-9335/a000325, Social Psychology, 49, 16-28 (2018).

Suárez Zozaya, M., Juventud de los Estudiantes Universitarios, https:doi.org/10.1016/jresu.2017.12.001, Revista de la Educación Sup., XLVI (4) (184), 39-54 (2017).

White, E., y Schulenberg, J., Academic Advising -A Focus on Learning, https:doi.org/10.1002/abc.20082, About Campus, 16 (6), 11-17 (2012). 
